\title{
INTRODUCTION TO TWISTED FACE-PAIRINGS
}

\author{
J. W. Cannon, W. J. Floyd, and W. R. Parry
}

\begin{abstract}
We give a mechanical recipe for creating simple face-pairing descriptions of closed 3-manifolds. We call the technique twisted face-pairing. Among the simpler twisted face-pairings we have studied, we have discovered manifolds (usually infinite classes of manifolds) which admit geometries based on $S^{3}, S^{2} \times \mathbf{R}$, $\mathbf{H}^{3}$, Solv, Nil, and the universal cover of $\operatorname{PSL}(2, \mathbf{R})$. Our work suggests, but does not resolve, the Conjecture: It is impossible because of the twisting involved in the construction to obtain, by twisted face-pairing, manifolds based on the product geometries $\mathbf{E}^{3}$ and $\mathbf{H}^{2} \times \mathbf{R}$. The twisted face-pairing technique is easily simple enough for use by students, and if used in conjunction with some software package like SnapPea, serves as a great exploratory playground in 3-manifold theory.
\end{abstract}

"The most important ingredient in any mathematical theory is a rich source of examples."

Mikhael Gromov

\section{Introduction}

A face-pairing $\epsilon$ of the 3-ball $B^{3}$ sews together the 2-cells of a cellulation of the boundary 2-sphere $S^{2}$ of $B^{3}$ isomorphically in pairs, every 2-cell being identified with a different 2-cell. This is done in such a way that the resulting quotient space $M=M(\epsilon)=B^{3} / \epsilon$ is naturally a cell complex. It is well known that every closed 3-manifold can be obtained as the quotient complex of some face-pairing.

The method of face-pairing has a defect. Namely, the quotient complex $M=$ $M(\epsilon)=B^{3} / \epsilon$ is always a closed 3-dimensional pseudomanifold, but it may fail to be a manifold because one or more of its vertices may have a link which is some closed 2-manifold other than the 2-sphere $S^{2}$. In fact, the following conjecture is surely true. Conjecture: If in the space of all face-pairings one is chosen at random, then with probability 1 the resulting quotient complex is not a manifold. Thus, although face-pairing descriptions of closed 3-manifolds lend themselves very naturally to the algorithmic construction of universal covers, associated Cayley graphs of $\pi_{1}$, and approximations to spaces at infinity, good examples are difficult to construct because they must either be discovered at random

Received January 26, 2000.

1991 Mathematics Subject Classification. 57Mxx.

Key words and phrases. 3-manifold constructions, surgeries on 3-manifolds, Thurston's geometries.

This research was supported in part by NSF research grants. 
(with probability 0) or deduced from other 3-manifold descriptions (Heegaard splittings, surgery descriptions, etc.).

The purpose of this paper is to informally report a construction which we discovered by accident as we were randomly exploring the barren landscape of face-pairings. Frustrated by our lack of success, we tried to modify our search according to our prior experience with knot spaces. We made four mistakes in pursuing the analogy, the avoidance of any one of which would have caused us to overlook the technique which we report here.

As the title indicates, this paper is only an introduction to twisted facepairing. Several other papers will follow beginning with [1] and [2]. Section 7 contains a brief discussion of further work.

We call our construction the method of twisted face-pairing. It takes as input an arbitrary orientation-reversing face-pairing $\epsilon$, as trivial and uninteresting as may be. (Every face of a cellulated $S^{2}$ inherits an orientation from any given orientation on $S^{2}$. The face-pairing reverses orientation if each face is sewn by an orientation-reversing isomorphism to its matched face.) As output the method supplies an infinite parametrized family of face-pairings, each having a closed, orientable 3-manifold as quotient. Even with trivial input, the output tends to be very interesting. The attendant geometry, fundamental group, first homology group, etc., are essentially always nontrivial even though the descriptions remain relatively simple.

We know of no technique which yields simpler face-pairing descriptions of interesting closed 3-manifolds, and certainly none which requires less effort. Among the simpler twisted face-pairings we have studied, we have discovered manifolds (usually infinite classes of manifolds) which admit geometries based on $S^{3}, S^{2} \times \mathbf{R}, \mathbf{H}^{3}$, Solv, Nil, and the universal cover of $\operatorname{PSL}(2, \mathbf{R})$. Without making an exhaustive search, but rather by trying some likely examples, we have obtained as twisted face-pairing manifolds approximately one fourth of the seventy-five manifolds with smallest volumes in SnapPea's [5] census of closed hyperbolic manifolds and also a few hyperbolic manifolds with relatively small volumes that were not on SnapPea's census. Although constructing twisted facepairings is easy, identifying the manifolds requires some effort. We postpone the presentation of examples to later papers after we develop a method to identify twisted face-pairing manifolds. Our work suggests, but does not resolve, the Conjecture: It is impossible because of the twisting involved in the construction to obtain, by twisted face-pairing, manifolds based on the product geometries $\mathbf{E}^{3}$ and $\mathbf{H}^{2} \times \mathbf{R}$.

The twisted face-pairing technique is easily simple enough for use by students, and if used in conjunction with some software package like SnapPea, serves as a great exploratory playground in 3-manifold theory. We have written two programs, pairsnap.c and partsnap.c, that provide input to SnapPea for twisted face-pairings and for partial twisted face-pairings respectively; they are freely available from http://www.math.vt.edu/people/floyd. 


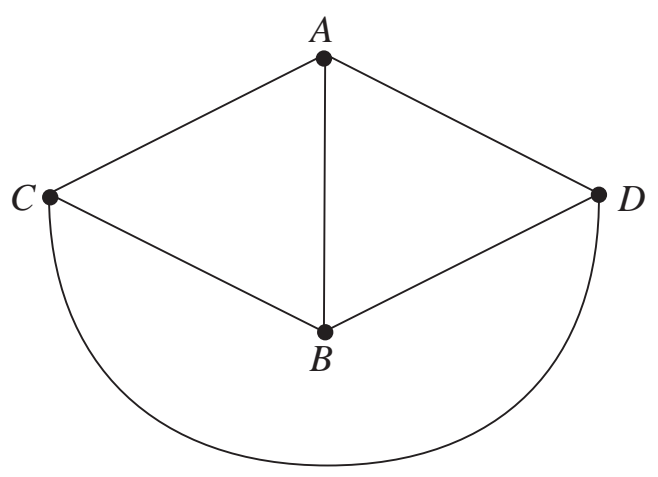

FiguRE 1. The tetrahedron $T$.

\section{The construction}

We describe the construction by example.

Step 1. The model face-pairing $\epsilon$. The method allows us to begin with an arbitrary orientation-reversing face-pairing $\epsilon$. For example, we realize the 3 -ball $B^{3}$ as a tetrahedron $T$ having vertices $A, B, C$ and $D$ as in Figure 1 . We pair faces in the most trivial of ways: we sew $A B C$ to $A B D$ by reflection in the common edge $A B$; we sew $A C D$ to $B C D$ by reflection in the common edge $C D$. We call this face-pairing the model face-pairing. It is not important whether or not the quotient pseudomanifold is a manifold. The quotient $M=M(\epsilon)=B^{3} / \epsilon$ which results from this particular model face-pairing is the 3 -sphere $S^{3}$. We summarize the sewings symbolically using permutation notation in the following way:

$$
\epsilon_{1}:\left(\begin{array}{ccc}
A & B & C \\
A & B & D
\end{array}\right) \quad \epsilon_{2}:\left(\begin{array}{ccc}
A & C & D \\
B & C & D
\end{array}\right)
$$

This means that face $A B C$ is identified with face $A B D$ by means of a homeomorphism $\epsilon_{1}$ which acts on vertices as indicated. Likewise face $A C D$ is identified with face $B C D$ by means of a homeomorphism $\epsilon_{2}$ which acts on vertices as indicated. We impose compatibility conditions on these homeomorphisms in Step 2. We call $\epsilon_{1}$ and $\epsilon_{2}$ face-pairing maps, and we set $\epsilon=\left\{\epsilon_{1}^{ \pm 1}, \epsilon_{2}^{ \pm 1}\right\}$.

Step 2. Edge cycles and edge cycle lengths $\ell$ of $\epsilon$. Since $\epsilon$ identifies faces in pairs, it follows easily that edges are identified in cycles. Our permutation notation for face-pairing maps is convenient for calculating these edge cycles without referring to Figure 1. Here are diagrams of the (three) edge cycles of our model face-pairing:

$$
\begin{gathered}
A B \stackrel{\epsilon_{1}}{\longrightarrow} A B \\
B C \stackrel{\epsilon_{1}}{\longrightarrow} B D \stackrel{\epsilon_{2}^{-1}}{\longrightarrow} A D \stackrel{\epsilon_{1}^{-1}}{\longrightarrow} A C \stackrel{\epsilon_{2}}{\longrightarrow} B C \\
C D \stackrel{\epsilon_{2}}{\longrightarrow} C D .
\end{gathered}
$$




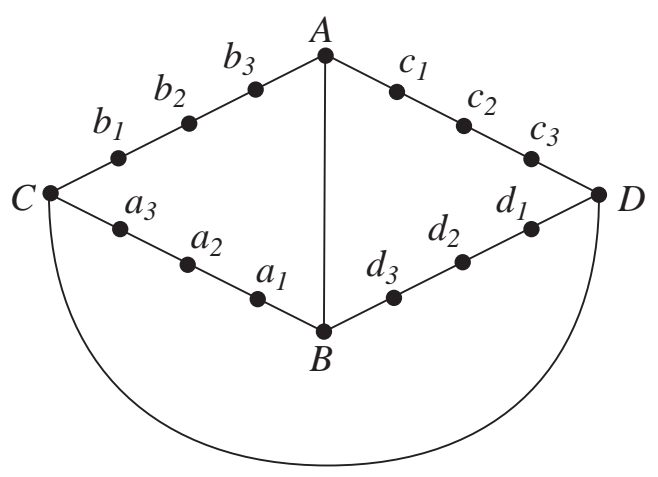

Figure 2. The subdivision of $T$.

The notation $B C \stackrel{\epsilon_{1}}{\longrightarrow} B D$, for example, indicates that the face-pairing map $\epsilon_{1}$ takes edge $B C$ to edge $B D$. We let $\ell_{i}$ denote the number of distinct edges in edge cycle number $i$, and we refer to $\ell_{i}$ as the length of edge cycle number $i$. We let $\ell=\left(\ell_{1}, \ell_{2}, \ldots, \ell_{k}\right)$ denote the corresponding tuple of integers. In our example, $\ell=(1,4,1)$.

As on page 123 of [4], to ensure that the quotient space $B^{3} / \epsilon$ is a cell complex, we require that our face-pairing maps satisfy the following face-pairing compatibility condition. Every edge cycle diagram as in line 2.1 in effect represents a composition of functions, these functions being face-pairing maps restricted to edges. We require that the composition of functions arising from every edge cycle diagram be the identity map. For example, for the second edge cycle diagram in line 2.1 we require that

$$
\left.\left.\left.\left.\epsilon_{2}\right|_{A C} \circ \epsilon_{1}^{-1}\right|_{A D} \circ \epsilon_{2}^{-1}\right|_{B D} \circ \epsilon_{1}\right|_{B C}
$$

be the identity map on $B C$. As in [4, Problem 3.2.10], it follows that the action of the face-pairing maps on vertices determines $B^{3} / \epsilon$ up to homeomorphism.

Step 3. Edge cycle multipliers $m$ and edge subdivision. Choose an arbitrary ordered set $m=\left(m_{1}, m_{2}, \ldots, m_{k}\right)$ of positive integers, one for each edge cycle. We call these positive integers edge cycle multipliers. The simplest possibility is of course $m_{i}=1$. For our example, we make that choice, so that $m=(1,1,1)$. Now, for each edge $e$ of edge cycle number $i$, subdivide $e$ into $\ell_{i} \cdot m_{i}$ subedges.

In our case, since $\ell_{1}=m_{1}=1$, we do not subdivide edge $A B$. Since $\ell_{2}=4$ and $m_{2}=1$, we subdivide each of $B C, B D, A D$, and $A C$ into $\ell_{2} \cdot m_{2}=4 \cdot 1=4$ subedges. Since $\ell_{3}=m_{3}=1$, we do not subdivide $C D$. See Figure 2. The face-pairing compatibility condition implies that we may choose this subdivision to be $\epsilon$-invariant. So, for example, $a_{1}$ is a new vertex in edge $B C$, and since the face-pairing map $\epsilon_{1}$ takes $B C$ to $B D$, we have $\epsilon_{1}\left(a_{1}\right)=d_{3}$.

Since we assume that our subdivision is $\epsilon$-invariant, it follows that $\epsilon$ induces a face-pairing, still denoted by $\epsilon$, which takes the subdivided cellulation to itself. 
The quotient pseudomanifold does not change. It is still $S^{3}$. We now write

$$
\begin{gathered}
\epsilon_{1}:\left(\begin{array}{lllllllll}
A & B & a_{1} & a_{2} & a_{3} & C & b_{1} & b_{2} & b_{3} \\
A & B & d_{3} & d_{2} & d_{1} & D & c_{3} & c_{2} & c_{1}
\end{array}\right) \\
\epsilon_{2}:\left(\begin{array}{lllllllll}
A & b_{3} & b_{2} & b_{1} & C & D & c_{3} & c_{2} & c_{1} \\
B & a_{1} & a_{2} & a_{3} & C & D & d_{1} & d_{2} & d_{3}
\end{array}\right) .
\end{gathered}
$$

Step 4. Twisting. In general we orient $B^{3}$; hence either the boundary of every face is oriented in the clockwise direction or the boundary of every face is oriented in the counterclockwise direction. For our example we orient the boundary of every face of our tetrahedron $T$ in the clockwise direction. Before applying the induced face-pairing maps, we twist each face one subedge in the direction of the orientation of the boundary. The result is a twisted face-pairing $\delta$. Of course we twist so that $\delta$ satisfies the face-pairing compatibility condition. This step completes the twisted face-pairing construction. For example, $\epsilon_{1}$ maps face $A B C$ to face $A B D$. Twisting $A B C$ one subedge in the clockwise direction takes the vertex $B$ to $a_{1}$, and $\epsilon_{1}\left(a_{1}\right)=d_{3}$. Thus $\delta_{1}(B)=d_{3}$. So for our example we have

$$
\begin{gathered}
\delta_{1}:\left(\begin{array}{ccccccccc}
b_{3} & A & B & a_{1} & a_{2} & a_{3} & C & b_{1} & b_{2} \\
A & B & d_{3} & d_{2} & d_{1} & D & c_{3} & c_{2} & c_{1}
\end{array}\right) \\
\delta_{2}:\left(\begin{array}{ccccccccc}
c_{1} & A & b_{3} & b_{2} & b_{1} & C & D & c_{3} & c_{2} \\
B & a_{1} & a_{2} & a_{3} & C & D & d_{1} & d_{2} & d_{3}
\end{array}\right),
\end{gathered}
$$

and $\delta=\left\{\delta_{1}^{ \pm 1}, \delta_{2}^{ \pm 1}\right\}$. The quotient after twisting is, as guaranteed by the theory of twisted face-pairings, a closed, orientable 3-manifold.

Caution. We emphasize that we obtain $\delta$ from $\epsilon$ by either always twisting clockwise or always twisting counterclockwise.

Main Theorem. If $\epsilon$ is an arbitrary orientation-reversing face-pairing, if $m=$ $\left(m_{1}, \ldots, m_{k}\right)$ is an arbitrary ordered set of positive integer multipliers, one for each edge cycle of $\epsilon$, and if $M=M(\epsilon, m)=B^{3} / \delta$ is the resulting twisted facepairing pseudomanifold, then $M$ is a closed, orientable 3-manifold. (Note that we need not mention the tuple $\ell$ since it is determined by $\epsilon$.)

The simple proof will be given in Section 4 after we have reviewed the necessary properties of Euler characteristic needed in the proof.

Our sample twisted face-pairing manifold is in fact a homology 3-sphere with infinite fundamental group which can be obtained by Dehn surgery on the figure eight knot. We shall be more precise about the relationship with the figure eight knot in Section 6, where we indicate how a modified version of twisted facepairing can be used to construct pseudomanifolds with controlled nonsphere vertex links. 
Here are diagrams of the (two) edge cycles for our sample twisted face-pairing:

$$
\begin{aligned}
b_{3} A \stackrel{\delta_{1}}{\longrightarrow} A B \stackrel{\delta_{1}}{\longrightarrow} B d_{3} \stackrel{\delta_{2}^{-1}}{\longrightarrow} c_{1} c_{2} \stackrel{\delta_{1}^{-1}}{\longrightarrow} b_{2} b_{1} \stackrel{\delta_{2}}{\longrightarrow} \\
a_{3} C \stackrel{\delta_{1}}{\longrightarrow} D c_{3} \stackrel{\delta_{2}}{\longrightarrow} d_{1} d_{2} \stackrel{\delta_{1}^{-1}}{\longrightarrow} a_{2} a_{1} \stackrel{\delta_{2}^{-1}}{\longrightarrow} b_{3} A \\
b_{1} C \stackrel{\delta_{2}}{\longrightarrow} C D \stackrel{\delta_{2}}{\longrightarrow} D d_{1} \stackrel{\delta_{1}^{-1}}{\longrightarrow} a_{3} a_{2} \stackrel{\delta_{2}^{-1}}{\longrightarrow} b_{2} b_{3} \stackrel{\delta_{1}}{\longrightarrow} \\
c_{1} A \stackrel{\delta_{2}}{\longrightarrow} B a_{1} \stackrel{\delta_{1}}{\longrightarrow} d_{3} d_{2} \stackrel{\delta_{2}^{-1}}{\longrightarrow} c_{2} c_{3} \stackrel{\delta_{1}^{-1}}{\longrightarrow} b_{1} C .
\end{aligned}
$$

Now we let $x_{1}$ and $x_{2}$ form a basis of a free group. We obtain words in this free group by replacing the $\epsilon$ 's and $\delta$ 's atop the arrows in our edge cycle diagrams by $x$ 's. From the model face-pairing $\epsilon$ the words are $x_{1}, x_{1} x_{2}^{-1} x_{1}^{-1} x_{2}$, and $x_{2}$. The following theorem asserts that these words give defining relators for $\pi_{1}\left(B^{3} / \epsilon\right)=$ $\pi_{1}\left(S^{3}\right)$. From the twisted face-pairing $\delta$ the words are

$$
x_{1} x_{1} x_{2}^{-1} x_{1}^{-1} x_{2} x_{1} x_{2} x_{1}^{-1} x_{2}^{-1} \quad \text { and } \quad x_{2} x_{2} x_{1}^{-1} x_{2}^{-1} x_{1} x_{2} x_{1} x_{2}^{-1} x_{1}^{-1} .
$$

These words likewise give defining relators for $\pi_{1}\left(B^{3} / \delta\right)$. In general, given a face-pairing $\epsilon$, let $M=B^{3} / \epsilon$ and let $M_{0}$ be the open manifold obtained by deleting the vertices from $M$. If $M$ is already a manifold, then $M$ and $M_{0}$ have the same fundamental group. Here is a classical theorem.

Theorem. The open manifold $M_{0}$ has fundamental group given by the generators and relators

$$
\left\langle x_{1}, x_{2}, \ldots \mid W_{1}, W_{2}, \ldots\right\rangle,
$$

where the generators $x_{1}, x_{2}, \ldots$ correspond to the face-pairing maps and the relators $W_{1}, W_{2}, \ldots$ are the words arising from the edge cycles as above.

Thus, for both our sample model face-pairing and twisted face-pairing, $M$ and $M_{0}$ have the same fundamental group, and the group presentations are the following:

$$
\left\langle x_{1}, x_{2} \mid x_{1}, x_{1} x_{2}^{-1} x_{1}^{-1} x_{2}, x_{2}\right\rangle
$$

and

$$
\left\langle x_{1}, x_{2} \mid x_{1}\left[x_{1}, x_{2}^{-1}\right]\left[x_{1}, x_{2}\right], x_{2}\left[x_{2}, x_{1}^{-1}\right]\left[x_{2}, x_{1}\right]\right\rangle,
$$

where the symbol $[a, b]$ denotes the commutator $a b a^{-1} b^{-1}$. The first group is obviously trivial. The second obviously has trivial abelianization.

\section{Euler characteristic of 3-dimensional pseudomanifolds}

In this section we recall a simple combinatorial proof of the following classical theorem which we learned from [3]. This theorem will be used in Section 4 to prove the main theorem. 
Theorem. Let $M$ be a triangulated closed pseudomanifold of dimension 3. Let $v_{1}, v_{2}, \ldots, v_{k}$ denote the vertices of $M$. Let $\operatorname{link}(\sigma, M)$ denote the link of a simplex $\sigma$ in $M$. Let $\chi$ denote Euler characteristic. Then

$$
2 \cdot \chi(M)=\sum_{i=1}^{k}\left[2-\chi\left(\operatorname{link}\left(v_{i}, M\right)\right)\right] \geq 0,
$$

with equality if and only if $M$ is a manifold.

Remark. Although the proof will make use of the triangulation of $M$, the result applies to any pseudomanifold arising from a face-pairing, for the pseudomanifold can easily be triangulated.

Proof. We will sum the Euler characteristics of the links of every simplex of $M$, dimension by dimension. We calculate the answers in two different ways. In the first, we use our knowledge that vertex links are 2-manifolds, edge links are 1-spheres, face links are 0-spheres, and 3-cell links are empty. In the second, we consider the contribution of each simplex to all link Euler characteristics and sum over all simplexes.

First calculation: Each 0-simplex (vertex) has link equal to a closed 2-manifold, and a closed manifold which is a connected sum of a 2 -sphere with $t$ tori and $p$ projective planes has Euler characteristic $2-2 t-p$. Each 1-simplex has link equal to a circle which has Euler characteristic 0. Each 2-simplex has link equal to a 0 -sphere (two points) which has Euler characteristic 2. Each 3-simplex has empty link. Let $C_{i}$ denote the number of simplexes of $M$ having dimension $i$. Then we have

$$
\begin{gathered}
\sum_{v \text { a vertex }} \chi(\operatorname{link} k(v))=\sum_{v \text { a vertex }}[2-2 t(v)-p(v)]=2 \cdot C_{0}-\sum_{v \text { a vertex }}[2 t(v)+p(v)], \\
\sum_{e \text { an edge }} \chi(\operatorname{link}(e))=\sum_{e \text { an edge }} 0=0, \\
\sum_{f \text { a face }} \chi(\operatorname{link} k(f))=\sum_{f \text { a face }} 2=2 \cdot C_{2}, \text { and } \\
\sum_{c \text { a } 3 \text {-cell }} \chi(\operatorname{link}(c))=\sum_{c \text { a } 3 \text {-cell }} 0=0 .
\end{gathered}
$$

Second calculation: We fix a simplex $\alpha$ of dimension $j$ and consider any $i$ dimensional face $\beta$ of $\alpha$, with $i<j$. Then there is a complementary face $\beta^{*}$ of $\alpha$ such that $\alpha$ is the join of $\beta$ and $\beta^{*}$. The dimension of $\beta^{*}$ is $j-i-1$, and $\beta^{*}$ is in the link of $\beta$ in $M$. We say that $\alpha$ contributes $(-1)^{j-i-1}$ to the Euler characteristic of the link of $\beta$. Note that there are exactly $\left(\begin{array}{l}j+1 \\ i+1\end{array}\right) i$-simplexes $\beta$ 
having link Euler characteristics to which $\alpha$ contributes. Thus we have

$$
\begin{gathered}
\sum_{v \text { a vertex }} \chi(\operatorname{link}(v))=C_{1}\left(\begin{array}{l}
2 \\
1
\end{array}\right)-C_{2}\left(\begin{array}{l}
3 \\
1
\end{array}\right)+C_{3}\left(\begin{array}{l}
4 \\
1
\end{array}\right), \\
\sum_{e \text { an edge }} \chi(\operatorname{link}(e))=C_{2}\left(\begin{array}{l}
3 \\
2
\end{array}\right)-C_{3}\left(\begin{array}{l}
4 \\
2
\end{array}\right), \text { and } \\
\sum_{f \text { a face }} \chi(\operatorname{link}(f))=C_{3}\left(\begin{array}{l}
4 \\
3
\end{array}\right) .
\end{gathered}
$$

If we sum and equate the two sides, we find

$$
\begin{gathered}
2 \cdot C_{0}+2 \cdot C_{2}-\sum_{v \text { a vertex }}[2 t(v)+p(v)]=2 \cdot C_{1}+2 \cdot C_{3}, \text { or } \\
2 \chi(M)=\sum_{v}[2 t(v)+p(v)] \geq 0 .
\end{gathered}
$$

Thus the Euler characteristic is 0 if and only if each $t(v)$ and each $p(v)$ is 0 if and only if each vertex link is a 2 -sphere. The theorem follows.

Exercise. Modify the proof to see that the Euler characteristic of any odddimensional simplicial manifold is 0 .

\section{Proof of the main theorem}

Let $\epsilon$ be a model face-pairing, $m$ an associated ordered set of positive integer multipliers, and $\delta$ the twisted face-pairing associated with $\epsilon$ and $m$. Let $M=$ $M(\epsilon, m)=B^{3} / \delta$ be the associated quotient complex. By the theorem on Euler characteristics in Section 3, in order to show that $M$ is a closed 3-manifold it suffices to show that the Euler characteristic $\chi$ of $M$ is 0 .

Let $C_{i}$ denote the number of $i$-cells that $M$ possesses. Then $C_{3}=1$, and $C_{2}$ is the number of face-pairs of the model face-pairing $\epsilon$. We shall complete the proof by showing that all the vertices of the subdivided $B^{3}$ are equivalent under $\delta$ so that $C_{0}=1\left(=C_{3}\right)$ and that there are exactly $C_{2}$ edge cycles of $\delta$ so that $C_{1}=C_{2}$. It will follow that $\chi=0$, so that $M$ is a closed manifold.

We next introduce some notation and terminology. Given a face $f$ of $B^{3}$, we let $f^{-1}$ denote the face with which $f$ is paired. We have face-pairing maps $\epsilon_{1}, \epsilon_{2}, \ldots$, one for every face-pair. We may assume that every face-pair of the subdivided $B^{3}$ has the form $\left\{F_{i}, F_{i}^{-1}\right\}$, where $\epsilon_{i}$ maps the face $F_{i}$ to the face $F_{i}^{-1}$, and so given $a \in\{ \pm 1\}$, we have that $\epsilon_{i}^{a}$ maps $F_{i}^{a}$ to $F_{i}^{-a}$. We refer to the vertices and edges of the original cellulation of $B^{3}$ as original vertices and edges of $B^{3}$. We refer to the edges of the subdivided $B^{3}$ as new edges, and we refer to the vertices of the subdivided $B^{3}$ which are not original vertices as new vertices. 


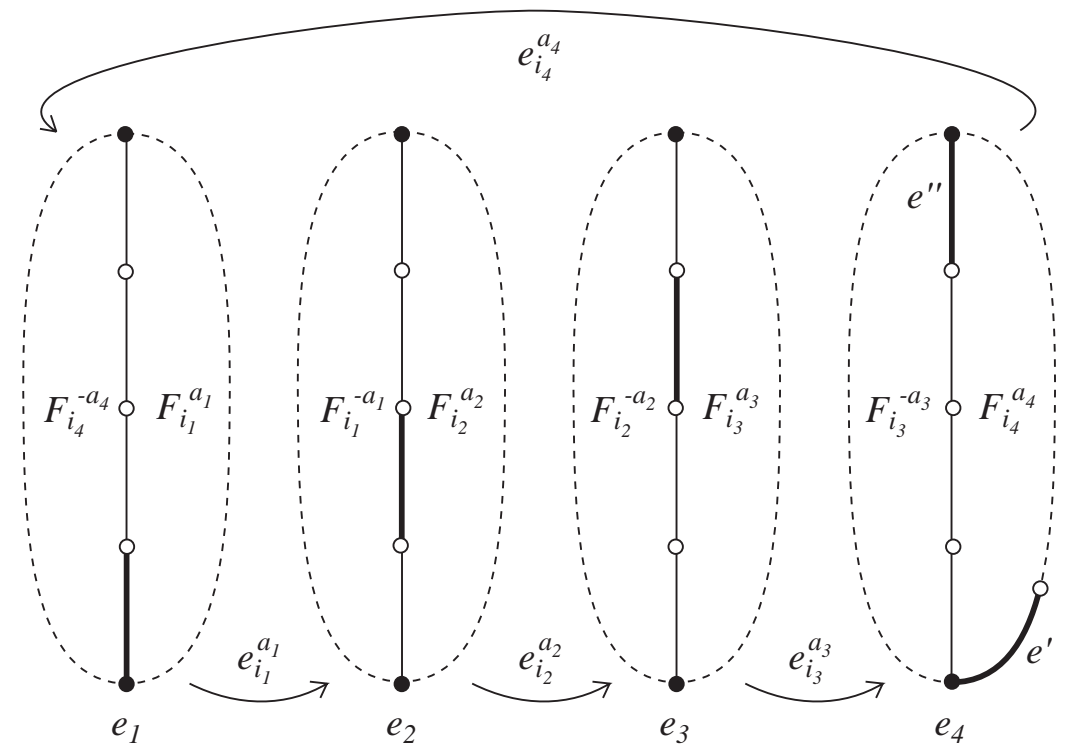

Figure 3. Part of the edge cycle of $e^{\prime}$ for $j=4$ and $k=1$.

The argument concentrates on a single edge cycle of the model face-pairing $\epsilon$ :

$$
e_{1} \stackrel{\epsilon_{i_{1}}^{a_{1}}}{\longrightarrow} e_{2} \stackrel{\epsilon_{i_{2}}^{a_{2}}}{\longrightarrow} \cdots \stackrel{\epsilon_{i_{j-1}}^{a_{j-1}}}{\longrightarrow} e_{j} \stackrel{\epsilon_{i_{j}}^{a_{j}}}{\longrightarrow} e_{1}
$$

where $e_{1}, \ldots, e_{j}$ are distinct original edges and every exponent $a_{1}, \ldots, a_{j}$ is \pm 1 . The number of edges in this edge cycle is $j$. Let $k$ be the multiplier associated with this edge cycle. Then each of the edges in this edge cycle is subdivided into $j \cdot k$ subedges in defining $\delta$.

Relative to the orientation of $F_{i_{j}}^{a_{j}}$, we concentrate on the new edge $e^{\prime}$ of $F_{i_{j}}^{a_{j}}$ which immediately precedes the original edge $e_{j}$. See Figure 3, where faces are oriented in the clockwise direction. Edges in the twisted face-pairing edge cycle of $e^{\prime}$ are drawn with thick arcs in Figure 3. For the twisted face-pairing $\delta$, edge $e^{\prime}$ is carried by $\delta_{i_{j}}^{a_{j}}$ to the first new edge of $e_{1}$ relative to $F_{i_{1}}^{a_{1}}$, thence onward by $\delta_{i_{1}}^{a_{1}}$ to the second new edge of $e_{2}$ relative to $F_{i_{2}}^{a_{2}}$, then to the third new edge of $e_{3}$ relative to $F_{i_{3}}^{a_{3}}$, and so forth, eventually to the $j$-th new edge of $e_{j}$ relative to $F_{i_{j}}^{a_{j}}$, and is passed around the given edge cycle of $\epsilon$ a total of $k$ times before it comes finally to the last new edge $e^{\prime \prime}$ of $e_{j}$ relative to $F_{i_{j}}^{a_{j}}$. It is as though $e^{\prime}$ has been moving systematically around the face $F_{i_{j}}^{a_{j}}$ from the new edge immediately preceding $e_{j}$, "along" the subdivided $e_{j}$, to the last new edge of $e_{j}$. In actuality, of course, it is jumping from edge to edge in the given edge cycle of $\epsilon$. But all is arranged so that it comes back to $F_{i_{j}}^{a_{j}}$ at exactly the correct time to begin a similar passage along the edge cycle of $\epsilon$ associated with the next original edge of $F_{i_{j}}^{a_{j}}$. This analysis when carried to its obvious conclusion precisely identifies the twisted face-pairing edge cycle of $e^{\prime}$. 
Now given a face $f$ of the subdivided $B^{3}$, we choose an original vertex $v$ of $f$, and to $f$ we associate the twisted face-pairing edge cycle of the new edge of $f$ which immediately precedes $v$. The above discussion shows that this edge cycle is independent of $v$. Furthermore, because of the twist and the reversal of orientation in pairing $f$ with $f^{-1}$, it follows that the twisted face-pairing edge cycle associated to $f^{-1}$ equals the twisted face-pairing edge cycle associated to $f$. This gives us a function from face-pairs of $B^{3}$ to twisted face-pairing edge cycles. From the above discussion it is easy to see that this function is bijective. Thus $C_{1}=C_{2}$.

The same argument shows that all of the original vertices of $B^{3}$ are equivalent under the twisted face-pairing $\delta$; indeed, the terminal endpoint of $e^{\prime}$ relative to

$F_{i_{j}}^{a_{j}}$ is identified with the terminal endpoint of $e^{\prime \prime}$ relative to $F_{i_{j}}^{a_{j}}$ so that adjacent original vertices are equivalent. But since the 1-skeleton of $B^{3}$ is connected, all original vertices are equivalent. Furthermore, every new vertex also obviously cycles to an original vertex. Hence $C_{0}=1$.

The proof that $\chi=0$ is therefore complete.

\section{More model face-pairings}

How does one easily construct model face-pairings?

It is an easy matter to construct all of the orientation-reversing face-pairings of a tetrahedron. By symmetry we may pair the faces in any way. We obtain nine face-pairings in a straightforward way. Some of these are equivalent to others by further symmetries. It turns out that there are four isomorphism classes of orientation-reversing face-pairings on a tetrahedron. Similarly, it is an easy matter to write down face-pairings with the other Platonic solids.

Having one face-pairing, one gets many more by invariant subdivision. The subdivision has no effect on the original quotient, but it changes the twisted face-pairings substantially.

For example, divide the 2-sphere into a northern and a southern hemisphere along the equator. Put some number of vertices on the equator. Then vertical projection from northern hemisphere to southern hemisphere is orientation reversing. Now subdivide the northern hemisphere in any way whatsoever and carry that subdivision to the southern hemisphere by projection. This construction supplies a huge number of trivial model face-pairings with very interesting twisted face-pairings.

Similarly, the antipodal map of the 2-sphere is orientation reversing. Subdivide via the equator. Subdivide the equator symmetrically under the antipodal map. Extend that subdivision to the northern hemisphere in any way whatsoever. Carry that subdivision to the southern hemisphere by the antipodal map.

One obtains interesting face-pairings by dividing the 2-sphere into an even number of digons joining north pole to south pole. One can pair these digons in many ways. 


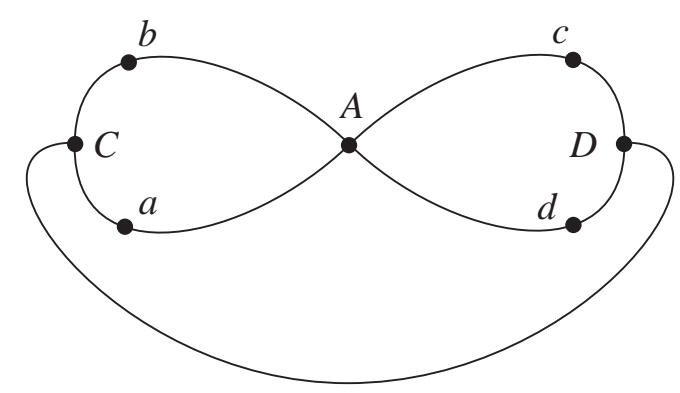

FigurE 4. The cellulation for the partial twisted face-pairing.

The important thing to realize is that no face-pairing is a bad face-pairing when it comes to the construction of twisted face-pairings.

\section{Partial twisted face-pairings}

By a slight modification of the twisted face-pairing construction we can construct pseudomanifolds with controlled vertex links. There are two ways of viewing the construction. We explain them here. But first we give an example.

This partial twisted face-pairing is based on our model above. The theory of partial twisted face-pairings is more complicated than that of twisted facepairings, and so we will give, but not explain until later, the dictated procedure in this case. We again begin with the tetrahedron in Figure 1. Proceeding according to formula, we collapse the edge $A B$ to a single vertex $A$ and we insert a single vertex into each of the four edges $A C, A D, B C$, and $B D$. See Figure 4. As in Step 3 of Section 2, there is an induced face-pairing before twisting still denoted by $\epsilon$ :

$$
\epsilon_{1}:\left(\begin{array}{cccc}
A & a & C & b \\
A & d & D & c
\end{array}\right) \quad \epsilon_{2}:\left(\begin{array}{ccccc}
A & b & C & D & c \\
A & a & C & D & d
\end{array}\right) .
$$

The partial twisted face-pairing $\partial$ twists only the second of these face identifications:

$$
\partial_{1}:\left(\begin{array}{cccc}
A & a & C & b \\
A & d & D & c
\end{array}\right) \quad \partial_{2}:\left(\begin{array}{ccccc}
c & A & b & C & D \\
A & a & C & D & d
\end{array}\right)
$$

where $\partial=\left\{\partial_{1}^{ \pm 1}, \partial_{2}^{ \pm 1}\right\}$. For both of these two face-pairings, the resulting pseudomanifold has one vertex whose link is a torus instead of a 2-sphere. If one removes the bad vertex from the untwisted quotient pseudomanifold, one obtains the uninteresting complement of an unknotted simple closed curve in $S^{3}$. The partial twisted face-pairing, on the other hand, has vertex complement homeomorphic to the complement of the figure eight knot in $S^{3}$. The partial twisted face-pairing is the simplest face-pairing description of the figure eight knot complement of which we are aware. 
First description of partial twisted face-pairing. Begin with a model face-pairing $\epsilon$ and form a twisted face-pairing $\delta$ associated with multiplier sequence $m$. Choose some collection $E_{1}, \ldots, E_{k}$ of edge cycles of $\delta$. Collapse each component of the union of all edges in $E_{1}, \ldots, E_{k}$ to a point. If the union does not separate the 2-sphere $S^{2}=\partial\left(B^{3}\right)$, then one obtains a new cellulation of $S^{2}$ and induced face-pairing $\partial$, which is the partial twisted face-pairing. If the union does separate $S^{2}$, then the collapse yields a new space $B^{\prime}$ which is not the 3 -ball $B^{3}$ but which is a $\mathrm{CW}$-complex with one 3 -cell. The boundary of $B^{\prime}$ is a wedge of 2 -spheres rather than a single 2 -sphere, but $B^{\prime}$ is perfectly suitable for a theory of (orientation-reversing) face-pairings. There is an induced facepairing $\partial$ in this case as well, and the induced face-pairing is the partial twisted face-pairing. We have the following theorem.

Theorem. If $k$ edge cycles are collapsed in forming a partial twisted face-pairing $\partial$ from a twisted face-pairing $\delta$, then the quotient complex $M(\partial)$ is a closed 3-dimensional pseudomanifold with one vertex. The link of that vertex is an orientable surface of genus $k$. If $M_{0}(\partial)$ denotes the open 3-manifold formed by deleting the one vertex from $M(\partial)$, then the fundamental group of $M_{0}(\partial)$ has presentation formed by deleting those relators from the presentation of $\pi_{1}(M(\epsilon, m))$ which correspond to the collapsed edge cycles.

Proof. Collapsing edges reduces the number of edge cycles by $k$. Hence the new $C_{1}$ is $C_{2}-k$. Collapsing edges cannot increase the number of vertex classes. Hence the new $C_{0}$ is still 1 . Hence the Euler characteristic is $k$. Since links are orientable in an orientable pseudomanifold, the link is a surface of genus $k$. Roger Vogeler pointed out to us the following way to see that this link is an orientable surface of genus $k$. We know that the 1-skeleton of $M(\epsilon, m)$ has one vertex and is isomorphic to a wedge of circles. In passing from $M(\epsilon, m)$ to $M(\partial)$ we collapse $k$ of the circles to a point. So the complement of the vertex in $M(\partial)$ is homeomorphic to the complement of the wedge of $k$ circles in $M(\epsilon, m)$. It easily follows that the link of the vertex of $M(\partial)$ is an orientable surface of genus $k$.

The fundamental group assertion follows from the theorem near the end of Section 2 ; the group relators mentioned vanish precisely because the corresponding edge cycles vanish.

Second description of partial twisted face-pairing. Partial twisted face-pairing can be described without passage through a complete twisted facepairing. One calculates the model face-pairing edge cycles as before. But one calculates the tuple $\ell$ of edge cycle lengths differently. In our first description of partial twisted face-pairings we choose a collection $E_{1}, \ldots, E_{k}$ of twisted facepairing edge cycles. The proof of the main theorem shows that this collection of edge cycles corresponds to a collection of faces; we have two faces $F_{j}$ and $F_{j}^{-1}$ for every edge cycle. In turn we have a corresponding collection of face-pairing maps with two face-pairing maps $\epsilon_{j}$ and $\epsilon_{j}^{-1}$ for every edge cycle. For a twisted face-pairing, one counts the number of face-pairing maps in the $i$-th edge cycle 
diagram to find $\ell_{i}$. For a partial twisted face-pairing, one counts only those face-pairing maps which are not from our chosen collection of face-pairing maps. It may happen now that some of the integers $\ell_{i}$ are 0 . Next subdivide each edge of $B^{3}$ into $\ell_{i} \cdot m_{i}$ subedges as before, where subdivision into 0 subedges indicates a total collapse of that edge to a point. There is an induced face-pairing on the new (possibly collapsed) complex. For those faces not in the collection of faces mentioned above, perform a twist as before. Those paired faces in our collection are to be sewn together without twisting.

Theorem. The second description of partial twisted face-pairing is equivalent to the first.

Proof. We return to the proof of the main theorem. As there we consider an edge cycle of the model face-pairing $\epsilon$ :

$$
e_{1} \stackrel{\epsilon_{i_{1}}^{a_{1}}}{\longrightarrow} e_{2} \stackrel{\epsilon_{i_{2}}^{a_{2}}}{\longrightarrow} \cdots \stackrel{\epsilon_{i_{j-1}}^{a_{j-1}}}{\longrightarrow} e_{j} \stackrel{\epsilon_{i_{j}}^{a_{j}}}{\longrightarrow} e_{1} .
$$

We constructed a bijection between the twisted face-pairing edge cycles and the set of face-pairs of $B^{3}$. In this bijection the twisted face-pairing edge cycle of the new edge of $F_{i_{j}}^{a_{j}}$ immediately preceding $e_{j}$ corresponds to the face-pair consisting of $F_{i_{j}}^{a_{j}}$ and $F_{i_{j}}^{-a_{j}}$. We saw that the new edge of $F_{i_{j}}^{a_{j}}$ which immediately precedes $e_{j}$ is mapped by the twisted face-pairing to the first new edge of $e_{1}$ relative to $F_{i_{1}}^{a_{1}}$, then to the second new edge of $e_{2}$ relative to $F_{i_{2}}^{a_{2}}$ and so on, and it eventually reaches the last new edge of $e_{j}$ relative to $F_{i_{j}}^{a_{j}}$. If the edge cycle in line 6.1 is the $p$-th model face-pairing edge cycle with multiplier $m_{p}$, then this accounts for $m_{p}$ new edges in each of the original edges $e_{1}, \ldots, e_{j}$. It follows that if $\epsilon_{i_{j}}^{ \pm 1}$ occurs $k_{i_{j}}$ times in line 6.1, then the twisted face-pairing edge cycle corresponding to $F_{i_{j}}^{a_{j}}$ and $F_{i_{j}}^{-a_{j}}$ contains $k_{i_{j}} m_{p}$ new edges in each of the original edges $e_{1}, \ldots, e_{j}$. This implies that for the partial twisted face-pairing, the number of edges into which each of the original edges $e_{1}, \ldots, e_{j}$ subdivides, as given by the first description, equals $m_{p}$ times the number of face-pairing maps in line 6.1 which are not from our chosen collection of face-pairing maps, in agreement with the second description.

Now let $f$ be a face of the new complex gotten from the original cellulation of $B^{3}$. If the pair of faces $f$ and $f^{-1}$ corresponds to one of the twisted facepairing edge cycles $E_{1}, \ldots, E_{k}$, then the last new edge of every original edge of $f$ collapses to a point. It is easy to see that this implies that the partial twisted face-pairing maps $f$ to $f^{-1}$ taking original vertices to original vertices. In other words, the partial twisted face-pairing maps $f$ to $f^{-1}$ without a twist. On the other hand, if the pair of faces $f$ and $f^{-1}$ does not correspond to one of the twisted face-pairing edge cycles $E_{1}, \ldots, E_{k}$, then the last new edge of every original edge of $f$ does not collapse to a point, and the partial twisted face-pairing maps $f$ to $f^{-1}$ with a twist.

This proves that the second description of partial twisted face-pairing is equivalent to the first. 
Our example from Section 2 gives rise to the example of this section. We collapse the edges in the first twisted face-pairing edge cycle in line 2.2. Recall from the end of Section 2 that the fundamental group of our twisted face-pairing manifold has presentation

$$
\left\langle x_{1}, x_{2} \mid x_{1}\left[x_{1}, x_{2}^{-1}\right]\left[x_{1}, x_{2}\right], x_{2}\left[x_{2}, x_{1}^{-1}\right]\left[x_{2}, x_{1}\right]\right\rangle .
$$

Given that the complement of the vertex of our partial twisted face-pairing pseudomanifold is the complement of the figure eight knot, then the first theorem of this section implies that the fundamental group of the figure eight knot has presentation

$$
\left\langle x_{1}, x_{2} \mid x_{2}\left[x_{2}, x_{1}^{-1}\right]\left[x_{2}, x_{1}\right]\right\rangle .
$$

We shall see elsewhere that the partial twisted face-pairing pseudomanifolds give rise to the complete twisted face-pairing manifolds by Dehn filling. Hence our homology sphere of Section 2 is obtained from the figure eight knot complement by Dehn filling.

\section{Questions and references to further work}

Our most tantalizing question is this:

Question 1. Which closed 3-manifolds are twisted face-pairing manifolds?

Question 1'. Which knot complements are partial twisted face-pairing manifolds?

In other papers we consider the following items.

(1) In [1] we investigate duality, homology, and fundamental groups of twisted face-pairing manifolds.

(2) In [2] we introduce ample twisted face-pairing manifolds, a combinatorially defined infinite subclass of all twisted face-pairing manifolds, and show that they all have Gromov hyperbolic fundamental groups and 2-spheres as spaces at infinity.

Question 2. If we assume Thurston's geometrization conjecture, then any given twisted face-pairing manifold will be associated with one or more of the Thurston geometries. Are there combinatorial criteria that allow us to recognize which geometries? Are there combinatorial criteria for recognizing twisted or partial twisted face-pairing manifolds whose groups are Kleinian (cocompact, finite volume, infinite volume)?

Question 3. Are there combinatorial or analytic relationships between twisted face-pairing manifolds and associated partial twisted face-pairing pseudomanifolds that have Kleinian structures?

(3) We combinatorially construct natural Heegaard diagrams of twisted facepairing manifolds. These diagrams suggest that twisted face-pairing manifolds might be thought of as a sort of integer lattice in the space of all closed 3manifolds. We also show that every irreducible Heegaard diagram has an underlying model face-pairing analogous to that in a twisted face-pairing. 
Question 4. Is there a fractional twisted face-pairing construction analogous to the method of twisted face-pairings that yields all 3-dimensional manifolds?

(4) We show that the Heegaard diagram of a twisted face-pairing manifold gives rise to a framed link in $S^{3}$ such that Dehn surgery on the framed link yields the given manifold. This allows us to explicitly identify many twisted face-pairing manifolds as lens spaces, more general Seifert fibered manifolds, Solv manifolds, etc.

(5) We give a survey of twisted face-pairing manifolds.

(6) We develop a general theory of face-pairings, including those which arise from partial twisted face-pairings. Among other things, we show how every facepairing pseudomanifold can be built up by surgery from simpler face-pairing pseudomanifolds by a process called edge constitution. Edge constitution requires one to find nonseparating curves in a closed 2-manifold given by edge pairing on a family of planar polygons.

Question 5. What is the simplest method for finding families of nonseparating curves in a closed 2-manifold given by edge pairing on a family of planar polygons?

\section{References}

[1] J. W. Cannon, W. J. Floyd, and W. R. Parry, Twisted face-pairing 3-manifolds, preprint.

[2] J. W. Cannon, W. J. Floyd, and W. R. Parry, Ample twisted face-pairing 3-manifolds, preprint.

[3] H. Seifert and W. Threlfall, Lehrbuch der Topologie, Chelsea Pub. Co., New York, 1947.

[4] W. P. Thurston, Three-Dimensional Geometry and Topology, Princeton Math. Ser. 35 (1997), Princeton University Press.

[5] J. Weeks, SnapPea: A computer program for creating and studying hyperbolic 3manifolds, available by anonymous ftp from geom.umn.edu/pub/software/snappea/.

Department of Mathematics, Brigham Young University, Provo, UT 84602, U.S.A.

E-mail address: cannon@math.byu.edu

Department of Mathematics, Virginia Tech, Blacksburg, VA 24061, U.S.A.

E-mail address: floyd@math.vt.edu

$U R L:$ http: //www.math.vt.edu/people/floyd

Department of Mathematics, Eastern Michigan University, Ypsilanti, Mi 48197, U.S.A.

E-mail address: walter.parry@emich.edu 